\title{
filosofía y niños: ¿para o con?
}

\author{
vania alarcón castillo ${ }^{1}$ \\ colegio jean le boulch \\ pontificia universidad católica del perú, perú \\ orcid id: https:/ / orcid.org/0000-0001-6849-4228
}

\section{resumen}

El presente artículo traza una comparación entre dos propuestas para introducir y ejercer la filosofía en espacios escolares que incluyan la participación de niños y niñas, estas son Filosofía para Niños $(F p N)$, programa diseñado principalmente por Matthew Lipman y Ann Sharp, y Filosofía con Niños $(F c N)$, la cual, de hecho, engloba un conjunto de (contra)propuestas de "segunda generación" -como las describen Vansieleghem y Kennedy (2011), en base a Reed y Johnson (1999)-, entre las que destacan las de Walter Kohan y Karin Murris, por mencionar algunas. El texto parte de algunos de los puntos de encuentro entre ambas para luego compararlas en cada una de sus dimensiones. Primero, se trata la propuesta de $F p N$. Segundo, la de $F c N$. Los puntos a discutir son sus ideas sobre la educación, la escuela y la educación filosófica, sus conceptos de infancia, el papel otorgado a los docentes y el vínculo establecido entre educación y política. Tercero, se detalla la crítica de $F c N$ a $F p N$ en relación con estos temas. Finalmente, el texto concluye con algunas ideas sobre la problemática de la introducción de la filosofía al espacio escolar. Particularmente, se apoya la propuesta de $F_{c} N$, fundamentalmente, debido a su coherente reconocimiento de la autonomía de los docentes y el elemento político de la educación, ya que la experiencia filosófica con niños es esencialmente cuestionadora, desafiante, $y$, por lo tanto, tiene la posibilidad de gestar transformaciones importantes, a nivel individualpersonal y también colectivo.

palabras clave: filosofía; educación; infancia; fpn; fcn.

\section{philosophy and children: for or with?}

\begin{abstract}
In this paper, two different philosophical proposals to introduce and carry out philosophy in school spaces which include the participation of children are compared. These are: Philosophy for Children (P4C), mainly developed by Matthew Lipman and Ann Sharp, and Philosophy with Children (PwC), which is actually a set of "second generation" (counter)proposals--as described by Vansieleghem and Kennedy (2011), based on Reed and Johnson (1999)--among which those created by Walter Kohan and Karin Murris, to mention a few, stand out. The text begins with some similarities between both proposals, before comparing them in each of their dimensions. First, $P 4 C$ is discussed. Second, PwC. Their ideas about education, school, philosophical education, their concept of childhood, the role given to teachers and their relation with politics are the main focus. Third, $P w C^{\prime} s$ critique of the $P 4 C$ program is studied. Finally, the paper concludes with some ideas on the issue of introducing philosophy in the school space. Particularly, PwC's proposal is supported, fundamentally because of its coherent acknowledgment of the autonomy of teachers and of the political element in education, and since philosophical experience with children is characterized in particular by questioning and critiquing, and, therefore offers the possibility of bringing about important transformations, at both the personal-individual and the collective levels,
\end{abstract}

keywords: philosophy; education; childhood; p4c; pwc.

\footnotetext{
${ }^{1}$ E-mail: valarcon@jlb.edu.pe
} 


\section{filosofia e crianças: para ou com?}

\section{resumo}

Neste artigo, são comparadas duas diferentes propostas filosóficas para introduzir e manter a filosofia em espaços escolares que incluem a participação de crianças. São elas: Filosofia para Crianças $(\mathrm{FpC})$, desenvolvida principalmente por Matthew Lipman e Ann Sharp, e Filosofia com Crianças $(F C C)$, que é na verdade um conjunto de (contra)propostas de uma "segunda geração" - como descrevem Vansieleghem e Kennedy (2011), baseados em Reed e Johnson (1999) -, entre as quais, aquelas criadas por Walter Kohan e Karin Murris, para mencionar algumas. O texto parte de alguns pontos de encontro entre as duas propostas, antes de compará-las em cada uma de suas dimensões. Primeiro, trata da proposta de FpC. Depois, da FCC. Os pontos de discussão são suas ideias sobre a educação, a escola e a educação filosófica, seus conceitos de infância, o papel outorgado aos professores e professoras e o vínculo estabelecido por cada uma dessas propostas entre educação e política. Então, detalha a crítica da $\mathrm{FcC}$ à FpC concernente a estes temas. Finalmente, o texto conclui com algumas ideias sobre a problemática da introdução da filosofia no espaço escolar. Particularmente, se apoia a proposta de $\mathrm{FcC}$, fundamentalmente, devido a seu coerente reconhecimento da autonomia dos professores e o elemento político da educação, já que a experiência filosófica com crianças é essencialmente questionadora, desafiante e, por isso, tem a possibilidade de gerar transformações importantes, num nível individualpessoal e também no nível coletivo.

palavras-chave: filosofia; educação; infância; fpc; fcc. 
filosofía y niños: ¿para o con?

\section{introducción}

Desde hace algunas décadas, la idea de introducir la enseñanza de la filosofía en las escuelas desde edades tempranas ha tomado mayor presencia en las discusiones sobre educación. Diferentes propuestas presuponen diferentes conceptos de filosofía, educación e infancia y, desde aquellos, responden a las dudas sobre la aplicabilidad o pertinencia de tal pretensión. Si la filosofía es una disciplina que se ocupa del sentido (Lipman, 2004, p. 18; Kohan y Waksman, 1999, p. 70; Kohan, 2016a, p. 69), entonces su vínculo con otras disciplinas es uno de cuestionamiento.

El vínculo entre filosofía y educación es particular, porque, ambas comparten una característica: no se restringen a una rama específica del conocimiento y su objeto respectivo, sino que son generales o totalizantes, a diferencia de otras disciplinas. Por ejemplo, la biología estudia la vida y los organismos vivos, la sociología se ocupa de lo social (sus prácticas, relaciones e instituciones), etc., en cambio, la filosofía y la educación se topan, en el camino de su reflexión, con diversos objetos de estudio. Las diferencia su forma de abordarlos y la dimensión de la que se ocupan.

Al explorar la historia, se puede ver que la educación siempre ha sido problemática y ha causado extrañeza, al verse llena de paradojas (Kohan, 2009, p. 112, Skliar, 2017, p. 41). Las paradojas recaen en dos puntos: la relación entre dependencia e independencia que supone el ejercicio educativo y la (im)posibilidad de educar. En el primer caso, se asume que el objetivo de la educación es formar personas, ciudadanos, que puedan desempeñarse en la sociedad, para lograr esto, se requeriría la guía de un grupo de personas competentes y la participación en una institución por un periodo prolongado de tiempo. En el segundo caso, el problema es si acaso -y cómo- se puede garantizar que aquello que se pretende enseñar sea de hecho lo aprendido por el educando. Esto nos remite al problema general de la comunicación humana: ¿qué nos garantiza que el otro entienda lo que queremos decir? Como bien señala Skliar, finalmente, "Entre el enseñar y el aprender hay un abismo, una distancia 
infinita", al punto que uno puede pretender igualdad a la hora de enseñar, pero no garantizarla a nivel de lo aprendido (Skliar, 2017, pp. 36, 32). Es decir, a pesar de nuestros esfuerzos como educadores para enseñar de una forma igualitaria, atendiendo a las diversas características de los educandos y adaptando nuestras formas y contenidos a las mismas, nada asegura que todos aprendan efectivamente lo mismo. Si se supera esta duda fundamental, la pregunta por cuál es la manera adecuada de educar a las personas también genera mucha polémica, y ha sido respondida de diversas maneras a lo largo de la historia. Como ilustra Cavell en su discusión sobre el aprendizaje de una lengua, la aproximación al problema de la educación ya viene cargada de muchos presupuestos que ocultan aquella experiencia que se trata de explorar (Cavell, 1979, p. 171).

Revisaré dos formas de articular una educación filosófica de la infancia: Filosofía para niños $(F p N)$ y Filosofía con niños $(F c N)$. Antes de pasar a detallar los puntos en común de las propuestas, prsento cada una de forma general. La diferencia entre ambas propuestas no se reduce al nombre o al uso de una u otra preposición, sino que implica preguntas sobre qué es la filosofía y cómo debería enseñarse (Murris, 2018, p. 1880). FpN es el programa desarrollado principalmente por Matthew Lipman y Ann Sharp desde los años 70 que se caracteriza por su uniformidad y carácter metódico, y que, a través de los años pudo extenderse a todas partes del mundo, con una recepción activa y crítica en muchos países. A partir de esta propuesta surge una "segunda generación" que abandona la concepción del programa como un método específico y pasa a considerarlo como un movimiento abierto a la diferencia, el cual abarca un conjunto de propuestas específicas (Vansieleghem y Kennedy, 2011, pp. 172, 177179; Reed y Johnson, 1999, pp. 60 ss.) $)^{2}$. Haynes y Murris sostienen que el elemento común dentro de esta variedad de propuestas de segunda generación

\footnotetext{
2 Otros de los exponentes y defensores de $F p N$ son Gareth Matthews, Leonard Nelson, Ekkehard Martens, etc., y entre la "segunda generación" también tenemos a David Kennedy, Michel Sasseville, Joanna Haynes, Oscar Brenifier, Marina Santi, etc. Vansieleghem y Kennedy ubican a Sharp en la segunda generación, pero debido a su estrecho vínculo con Lipman y su papel en la diseminación del programa de $F p N$, opto por agruparla dentro de esta primera propuesta (2011, pp. 172-177).
} 
es el “consenso acerca de la 'comunidad de investigación' como la pedagogía de FcN" (Haynes y Murris, 2013, pp. 299-300, n. 1). Discuto este tema más adelante.

La segunda generación no es del todo opuesta a la primera, ni mucho menos un ataque hacia la misma, sino, como mencionan Vansieleghem y Kennedy, "un paso necesario" frente al cambio de contexto educacional y global, esto es, una "autocorrección" (2011, p. 177). Estas propuestas son las que se denominan con el rótulo de $F c N$. El cambio de preposición en el nombre es, entonces, una forma de la "segunda generación" de distinguirse del programa “oficial" de FpN (Murris, 2016, p. 64). De hecho, esta distinción fue hecha a pedido de Lipman, "por el bien de la claridad" y evitar confundir su programa oficial con estas nacientes alternativas (Haynes y Murris, 2013, pp. 299-300, n. 1).

Ahora, ¿qué implicaciones tiene este cambio de nombre? La diferencia entre el empleo de la proposición "para" y "con" denota una relación diferente entre la filosofía, los adultos que la enseñan y los niños. En el primer caso, se trata de una relación externa, jerárquica, asimétrica, incluso paternalista, la cual sugirie que los adultos otorgan algo a los niños; en el segundo, una relación interna, colaborativa y abierta, que enfatiza el elemento dialógico y que la filosofía es algo que se practica en conjunto (Kohan, 2016b, pp. 127-128; Vansieleghem y Kennedy, 2011, pp. 178-179, 181, n. 1).

Kohan sostiene que:

El "para" no es de las preposiciones más interesantes por la distancia y exterioridad que establece entre lo que pone en relación, en este caso la filosofía y la infancia. Incluso "Filosofía" como sustantivo tampoco es tan propicio si pensamos en una disciplina o saber cuando se trata más bien de una actividad. Y "niños", aunque la " $x$ " mejora un poco el problema de género, tampoco es una palabra sugerente (Kohan en SánchezManjavacas, 2020, pp. 111-112).

Por eso propone, como alternativa, el rótulo: “Filosofar con (o entre) chicxs".

Habiendo presentado esta imagen general, empezaré señalando algunos de los puntos de encuentro entre ambas propuestas para luego diferenciarlas en cada una de sus dimensiones. Primero, trataré la propuesta de FpN y en seguida a la de $F_{c} N$. Me detendré a estudiar sus ideas sobre la educación, la escuela y la educación filosófica, sus métodos, conceptos de infancia, el papel otorgado a los 
docentes y el vínculo establecido entre educación y política. Posteriormente, revisaré la crítica de $F_{c} N$ a $F p N$ en relación con estos mismos puntos. Por último, concluyo con algunas de mis ideas sobre la problemática de la introducción de la filosofía al espacio escolar. Particularmente, me alineo la propuesta de $F_{c N}$ debido a su coherente reconocimiento de la autonomía de los docentes y el elemento político de la educación. Sostengo que la experiencia filosófica con niños es esencialmente cuestionadora, desafiante, $y$, por lo tanto, tiene la posibilidad fundamental de gestar transformaciones importantes, a nivel individual-personal y también colectivo.

\section{1. algunos puntos en común}

Ambas propuestas se posicionan contra cierta forma de entender la educación, esto es, su reducción a la transmisión de conocimiento (Lipman, Sharp y Oscanyan, 1992, pp. 113-114; Lipman, 2016, p. 11; Kohan y Waksman, 1999, p. 17; Kohan, 2016b, p. 156; Kohan y Olarieta, 2013, p. 40) y en la que se la suele vincular a la proyección de objetivos tales como la superación de un estado de minoría de edad, heteronomía e incapacidad, que requieren de la guía de otro ilustrado, capaz, el que sabe (Kohan, 2010, p. 266). En efecto, los contenidos transmitidos serían condición del logro de aquellos objetivos. Frente a este retrato, las propuestas que revisaré defienden que enseñar filosofía a niños no implica la transmisión de contenidos específicos (esto es, historia de la filosofía), sino la recreación de la práctica misma de la filosofía con ellos en el espacio de la escuela, desde sus propios intereses, interrogantes y reflexiones.

Ligado a esto, otro punto en común es la centralidad del diálogo en ambas propuestas. En efecto, el “hacer filosofía” se entiende como un ejercicio dialógicocolectivo. Pero, como se verá, hay un matiz particular en cada una de ellas. En el caso de $F p N$, este diálogo es entendido como un método bien delineado, proponen una comunidad de investigación con una estructura de momentos claramente diferenciados, en base a materiales específicos (Lipman, Sharp y Oscanyan, 1992, pp. 140-141). En cambio, en el caso de $F c N$, se lo entiende de forma más abierta, y se defiende que su composición específica y materiales sean determinados por los maestros involucrados en la experiencia, y no de forma 
previa y estándar, por un programa oficial (Kohan, en Sánchez-Manjavacas, 2020, p. 121; Kohan, 2010, p. 62).

Asimismo, debido al énfasis en los procesos y ejercicios antes que los contenidos transmitidos y resultados, en ambas propuestas se reconoce que el aprendizaje de los estudiantes está íntimamente relacionado no solo al discurso de los maestros, sino sus acciones y comportamiento, que funcionan como fuente de motivación (Lipman, 2004, pp. 14, 133; Lipman, Sharp y Oscanyan, 1992, pp. 170-171; Kohan, en Sánchez-Manjavacas, 2020, pp. 113; Kohan, 2009, p. 81). Así, ambas, a su manera, desafían la imagen de un maestro cuyo conocimiento ya está acabado, independiente del proceso realizado en el aula con sus alumnos (Kohan, 2010, pp. 46, 50). Lipman diría que el docente considera o tiene cierta apertura respecto a sus alumnos, sus intereses y perspectivas (Lipman, Sharp y Oscanyan, 1992, p. 118). En cambio, Kohan sugiere que el maestro aprende mientras enseña: el mundo le resulta tan sorprendente como a sus alumnos, estando dispuesto a enrumbarse con ellos en una experiencia filosófica, la cual es inherentemente transformadora (Kohan y Olarieta, 2013, p. 39; Wozniak, 2013, pp. 137-138).

En lo presentado se aprecia que ambas propuestas, en la dimensión práctica pueden ser, hasta cierto punto, conciliables. Pero es necesario subrayar que parten de premisas de carácter político distantes, lo que le da un espíritu particular a cada una, como se propone a continuación.

\section{Filosofía para niños}

\subsection{Filosofía y educación: medios y fines}

Como sostiene Kohan, "El aporte sustantivo y específico de Lipman consiste en el papel que atribuye a la filosofía en la experiencia educativa y en su idea ciertamente revolucionaria de que los niños son aptos para practicar la filosofía tan pronto como comienza su educación institucionalizada" (Kohan y Waksman, 1999, p. 21). Lipman y Sharp fundamentan el vínculo filosofíaeducación en el esencial carácter normativo de la filosofía y su papel para desarrollar las habilidades cognitivas que constituyen lo que denominan el "pensamiento multidimensional", en tanto su ejercicio implica elementos conceptuales, epistémicos, críticos, pero también creativos y valorativos 
(Lipman, Sharp y Oscanyan, 1992, p. 114; Lipman, 2004, p. 113). Específicamente, tal pensamiento multidimensional consiste en la articulación de tres dimensiones: pensamiento crítico, creativo y cuidadoso (caring), que corresponden a diferentes formas de investigación (Lipman, 2016, p. 11). Lo novedoso de la propuesta de Lipman y Sharp es que articulan y equilibran la educación de las emociones y del pensamiento con una orientación práctica, dándoles la misma importancia a ambos, a diferencia del énfasis usual dado al mero pensamiento crítico en propuestas educativas más tradicionales (Lipman, 2016, pp. 12, 13, 14; Kohan y Waksman, 1999, pp. 19-20). La importancia del desarrollo de este pensamiento radica en su vinculación con los ideales de razonabilidad y, democracia y la mejora académica global en el ámbito escolar que genera, es decir, no se restringe al curso de filosofía (Lipman, 2016, p. 30).

Es decir, $F p N$ concibe la filosofía como herramienta educativa, como medio para el desarrollo de tal pensamiento (Lipman, Sharp y Oscanyan, 1992, pp. 117, 123). No obstante, el fin último es fomentar el desarrollo de ciudadanos razonables y democráticos (Lipman, 2016, p. 15). Llama la atención que Lipman y Sharp cuestionen la premisa individualista del liberalismo, mas no estas otras dos. Democracia y razonabilidad son consideradas "ideas regulativas" de la estructura social y el carácter (individual y colectivo), respectivamente (Lipman, 2016, pp. 15, 38). La persona razonable demuestra coherencia entre sus juicios y acciones, puede dialogar con otros, expresando y defendiendo sus ideas, escuchando a los otros y sus posibles críticas de forma respetuosa, es abierta al efecto de estas y, consecuentemente, a la modificación de su perspectiva (Sharp y Splitter, 1995, pp. 22-23, Lipman, Sharp y Oscanyan, 1992, p. 121). La razonabilidad excede la mera racionalidad: esta última "es el pensamiento crítico, la lógica formal e informal, con sus habilidades de investigación, de formación de conceptos, traducción y raciocinio", en cambio la racionalidad "es el pensamiento de orden superior: pensamiento crítico, creativo y cuidadoso" (Kohan, 2020, p. 225).

Entonces, la intención de esta propuesta consiste en reformar el sistema educativo dentro del marco institucional-democrático existente, promoviendo el desarrollo de un tipo de pensamiento que permite la realización de las ideas 
regulativas de democracia y razonabilidad (Sharp y Splitter, 1995, p. 36; Kohan y Waksman, 1999, p. 17). Lipman y Sharp reconocen el carácter instrumental de su propuesta y lo justifican alegando que es la única alternativa para lograr la introducción de la filosofía en el currículo escolar: "Si la filosofía es admitida en el curriculum en las condiciones actuales, será únicamente si puede demostrar a los que dirigen las escuelas que puede introducir diferencias significativas en la conducta global del niño". De ahí su énfasis en los beneficios de la enseñanza de la filosofía en otras áreas de aprendizaje, en tanto tiene efectos en la lectura, el razonamiento, la creatividad y actitud del niño frente a sí mismo, la escuela y sus compañeros (Lipman, Sharp y Oscanyan, 1992, pp. 116-118). Sin embargo, sugieren que, de hecho, la filosofía "es un área humanística cuyo aprendizaje representa un enriquecimiento que no necesita ninguna otra justificación" (Lipman, Sharp y Oscanyan, 1992, p. 117). Así, el tinte instrumental de la propuesta sería una estrategia para garantizar su inserción en el sistema educativo, no su elemento esencial: “admitamos la posición de los responsables educativos y padres más desconfiados: si la filosofía para niños no es buena educación, no tiene un sitio en las escuelas. El peso de la prueba cae entonces en el programa mismo para demostrar las diferencias que puede introducir en los estudiantes a los que se les imparte" (Lipman, Sharp y Oscanyan, 1992, p. 123).

\section{2 el método de fpn}

Para garantizar el desarrollo del pensamiento multidimensional, Lipman y Sharp diseñan, basándose principalmente en la filosofía pragmatista americana, una pedagogía: la comunidad de investigación o indagación, cuya epistemología es un equilibrio reflexivo -de ahí su vínculo con la democracia(Lipman, 2016, p. 10). De cierta forma, la comunidad de investigación es, a su vez, condición e ideal de la práctica de la filosofía en la escuela (Lago, 2006, p. 58). Esto debido a que la idea detrás de la comunidad de investigación es promover un ambiente en clase que permita el desarrollo de las habilidades que componen el pensamiento multidimensional (Sharp y Splitter 1995: 36). Se reconoce que el desarrollo de tal pensamiento excede la transmisión de un contenido discursivo: tiene que encarnarse en la práctica, metodología y 
ambiente de las aulas de clase (Sharp y Splitter, 1995, p. 36). Por lo tanto, estas comunidades se caracterizan por la centralidad del diálogo abierto y democrático, en el que se formulan problemas y se articulan con otras preguntas y respuestas, así como por el énfasis en el proceso por sobre los resultados de la indagación, razón por la cual no se busca necesariamente un consenso, sino que se aprecia el disenso (Lipman, Sharp y Oscanyan, 1992, pp. 206-208),

Los temas de investigación de la comunidad de indagación son estimulados por los materiales que conforman el programa diseñado por Lipman y Sharp y, se supone, han de corresponder a los intereses de los alumnos (Lipman, Sharp y Oscanyan, 1992, pp. 140-141). Otra de sus características es el elemento de la metacognición: lo que determina el tipo del diálogo correspondiente al pensamiento multidimensional es la capacidad de referirse no solo a determinados contenidos sino también al diálogo y reflexión mismos: por ejemplo, respecto a la ética, no basta que los niños puedan sostener juicios éticos, sino que los reconozcan como tales, identifiquen las creencias y valores sobre las que se basan, su expresión en el diálogo, y, así, mejoren sus justificaciones (Lipman, Sharp y Oscanyan, 1992, p. 121). Esto se relaciona al vínculo entre los procedimientos o formas, y los contenidos discutidos: "Consequently, when this process is internalized or introjected by the participants, they come to think in moves that resemble its procedures. They come to think as the process thinks" (Lipman, 2003, p. 20).

\section{3 los maestros y los materiales}

Lipman sostiene que los elementos necesarios del programa son los maestros, la pedagogía, la comunidad de investigación y el currículum (esto es, el conjunto de textos usados en las comunidades) (Lipman, 2008, p. 49). Hace falta discutir sobre los maestros y los textos. Para garantizar los fines de su proyecto, Lipman y Sharp diseñaron un programa exhaustivo, acompañado de un material de uso recomendado -aunque eran receptivos frente a la idea del uso de otros materiales, siempre y cuando formen parte de algún programa preparado para esos fines-. La decisión de diseñar las lecturas del curriculum se basa en que, según Lipman, las historias para niños no son las más adecuadas 
para lograr sus fines propuestos, ya que en estas se suelen dar retratos unidimensionales de los personajes, que no muestran un pensamiento multidimensional desarrollado (Lipman, Sharp y Oscanyan, 1992, p. 356). Lipman vuelve protagonistas de sus historias precisamente a este tipo de niñospersonajes, para que cumplan un rol de modelos para los niños que participan de $F p N$. Esto es, el texto no es considerado un fin en sí mismo, sino un medio, lo cual reduce la literatura a su valor instrumental (Kohan y Waksman, 1999, pp. $34,54)$.

Respecto a los docentes, en el programa de Lipman y Sharp no está contemplado que sean filósofos, sino que se espera que provengan de otras disciplinas: de ahí la vital importancia en la capacitación y el control del material para garantizar el ejercicio dialógico de la comunidad de investigación (Lipman, Sharp y Oscanyan, 1992, p. 120; Kohan y Waksman, 1999, pp. 4-5). En su actuar, los docentes también deben exhibir un ejercicio articulado de las dimensiones crítica, creativa y ética (cuidante) del pensamiento multidimensional, mediante su respeto de las formas o procedimientos de investigación y mostrando atención a que los estudiantes también los respeten (Lipman, Sharp y Oscanyan, 1992, p. 118). Para esto, el material propuesto por Lipman y Sharp consiste en una serie de recomendaciones, ejercicios, preguntas, dinámicas, etc., cuidadosamente diseñados para generar un acercamiento orgánico entre los niños y la filosofía, progresivo en "profundidad, amplitud y sofisticación" (Lipman, 2004, p. 68; Lipman, Sharp y Oscanyan, 1992, pp. 167, 189 ss.). La idea de trasladar la responsabilidad de la mediación entre la filosofía y los niños de los maestros al programa (curriculum y materiales) -o, en todo caso, de distribuirla entre ambos- es, según Lipman, una forma de aliviar la carga de los maestros (2004, pp. 33-34).

Gran parte de las objeciones de Kohan a $F p N$ se orientan contra papel mecánico y externo al cual los docentes son reducidos, en tanto no participan de la formulación de los programas, sino que solo los aplican (Kohan y Waksman, 1999, p. 27; Kohan, 2020, pp. 211-226). Otra de sus críticas se centra en esta instrumentalización de las disciplinas, defendiendo una relación horizontal entre ellas, como se desarrolla en detalle más adelante. 


\section{4 la infancia}

Lipman y Sharp se muestran críticos ante la concepción tradicional de la infancia, la cual identifica al niño con una carencia de razón o pensamiento complejo, de lenguaje, de experiencia de vida, cuya mente sería un mero recipiente vacío a ser llenado, educado en la escuela para desarrollarse como un adulto razonable (Santiago, 2006, p. 33). Piaget, por ejemplo, sostiene que los niños carecen del pensamiento complejo necesario para ejecutar un pensamiento filosófico (1951, pp. 7, 10, 37-39, 43-44, 110-111, 166-168). Según la propuesta de $F p N$, el problema no está en la real capacidad de los niños para hacer filosofía, sino en nuestra forma adulta de medirla, la cual se suele enfocar en su uso del lenguaje, en la “capacidad de responder preguntas más que por la capacidad de plantearlas, [...] la resolución de problemas más que de competencia en su reconocimiento y formulación", al punto que "hasta que los niños no ven la realidad como los adultos, se ignora y desprecia de forma sistemática la riqueza y finura de sus puntos de vista sobre el mundo" (Lipman, Sharp y Oscanyan, 1992, p. 138). Matthews, otro defensor de $F p N$, sostiene que Piaget está tan enfrascado en tratar de clasificar las respuestas de los niños en las etapas del desarrollo del pensamiento que se vuelve insensible ante la perplejidad filosófica que la experiencia del pensamiento en los niños revela (2014, pp. 73-74).

Frente a este retrato, $F p N$ resalta la capacidad de los niños para desarrollar un pensamiento filosófico, además de su curiosidad, sosteniendo que estos ya piensan de forma abstracta y no meramente concreta, lo cual se aprecia en sus interacciones (por ejemplo, la insistencia en las preguntas por el porqué de las cosas) y que, además, pueden reflexionar sobre ciertos conceptos filosóficos (como la mente, verdad, causalidad, etc.) cuando son adecuadamente vinculados a sus intereses y experiencias (Sharp y Splitter, 1995, pp. 27, 41; Lipman, Sharp y Oscanyan, 1992, p. 135). La presuposición de la propuesta de FpN es que "todos los niños tienen interés y capacidad para realizar tal actividad [la filosofía]" y que "hay formas de implicar a los niños en el repertorio filosófico" (Lipman, Sharp y Oscanyan, 1992, p. 114). Pero es necesario considerar que el hecho de que los niños se muestren capaces de razonar, no significa automáticamente que ejerzan una actividad filosófica espontánea (Lipman, Sharp y Oscanyan, 1992, p. 135). 
Justamente, el programa trazado posibilitaría la transformación del aula en un ambiente propicio "que saca a la palestra los temas que son relevantes para los problemas de los niños, temas que son suficientemente variados como para que la invitación [...] alcance también a temas contemplativos y creativos" (Lipman, Sharp y Oscanyan, 1992, 140-141). Sin embargo, ciertos elementos de la propuesta hacen que no se aleje demasiado de la concepción tradicional de la infancia.

Recordemos que el programa de $F p N$ está planteado como una forma de desarrollar el pensamiento multidimensional en los niños para, de esa manera, formar ciudadanos que encarnen los ideales de razonabilidad y democracia. Se puede ver el énfasis del programa en el desarrollo o crecimiento en la siguiente cita: “Si [...] resulta que gracias al programa se ha elevado la sensibilidad y el juicio, es muy probable que el programa haya servido no simplemente para acelerar el crecimiento de los niños, sino para ampliar su real capacidad de crecer" (Lipman, Sharp y Oscanyan, 1992, p. 146). Esto resalta una vez más el carácter instrumental otorgado a la filosofía en esta propuesta. En ese sentido, si bien toman en cuenta algunas capacidades que la consideración tradicional de la infancia niega, se sigue presuponiendo la idea de que el ser humano se desarrolla linealmente a través del tiempo y, por lo tanto, su educación es vital para tal desarrollo o crecimiento (Kohan, 2009, pp. 15-16). Aquella imagen es lo que se conoce como una mentalidad de desarrollo (developmentalism), la cual es criticada por numerosos autores, entre ellos Murris, quien sugiere que esta mentalidad suele esencializar y generalizar al niño, sus capacidades y potencialidades, limitando nuestra capacidad para pensar en las posibilidades de la educación (2013, pp. 253-254). Esta mentalidad postula como objetivos de tal desarrollo individuos intelectualmente flexibles, capaces de resolver problemas e interactuar con otros, resilientes y adaptables a contextos de economías altamente competitivas en constante cambio, es decir, individuos que puedan integrarse y servir al modelo imperante (Murris 2019: 58); el ideal de desarrollo perseguido en $F p N$ se alinea al modelo democrático-neoliberal, en vez de cuestionarlo. 


\section{5 el elemento político}

Ya al discutir sobre el método de $F p N$ y su concepto de infancia se ha podido observar cómo el elemento político atraviesa toda la propuesta. Más allá de la pretensión de neutralidad, es innegable el carácter político de la educación, en tanto la polis se compone de individuos, en cuyas vidas la educación es un elemento clave, que influye en la construcción de su identidad, creencias, modos de comportamiento, ideales, etc. Lipman y Sharp insisten en que enseñar filosofía no implica indicarles a los niños valores o normas éticas (contenidos), sino que el énfasis del programa está en familiarizarlos con la forma de investigación, con una preocupación por la justificación de sus propios juicios (Lipman, Sharp y Oscanyan, 1992, p. 145). Sin embargo, paralelamente, insisten en la importancia de los ideales de democracia y razonabilidad como normas regulativas de la comunidad de investigación (Lipman, 2016, p. 15). Entonces, el fin propuesto por $F p N$, promover el desarrollo del pensamiento multidimensional, y de esta forma, individuos que encarnen los ideales de democracia y razonabilidad, implica, a su vez, el fin más amplio de desarrollar una sociedad que encarne los mismos. En la sección 4.4 discutiré las presuposiciones y consecuencias de esto.

\section{3. filosofía con niños}

\section{1 la educación y filosofía como experiencias}

Del lado de las propuestas de $F_{c} N$, se rescata el valor intrínseco de la filosofía y su vínculo con la educación. La reflexión filosófica no es vista como una herramienta o competencia a desarrollar, sino un acontecimiento que se mide por los sentidos, posibilidades y la alegría que se desprenden del proceso mismo de búsqueda de sentido, motivado por el cuestionamiento de lo dado y la proyección de sus posibilidades, lo cual implica un intercambio con los otros (Kohan, 2009, p. 65; Kohan y Waksman, 1999, p. 70). Kohan esboza el pensamiento como una experiencia, siguiendo el concepto propuesto por Foucault, como "algo de lo que se sale transformado", un desplazamiento orientado a lo desconocido, un acontecimiento "que arranca al sujeto de sí mismo", y que, como tal, no puede ser anticipado (Trombadori, 2010, pp. 43, 52). 
Masschelein y Simons presentan una imagen interesante para vincular esta consideración sobre la experiencia del pensamiento a la educación: la escuela como medio puro, sin fin ni destino predeterminado, pero como aquello que "hace posible todos los destinos" (2014, p. 38). La educación, así, tendría que enfocarse en la apertura de una experiencia particular presente, otorgarle a la persona "una cierta salida de sí misma en dirección al mundo" y sus posibilidades, su multiplicidad, antes que la proyección y anticipación de resultados específicos futuros (Kohan, 2004, p. 276; 2009, pp. 24-25; 2016, p. 78; Santiago, 2006, p. 33; Kohan y Waksman, 1999, pp. 26). Desde la perspectiva de Kohan, el objetivo de la educación no es sino la problematización de la propia vida individual y social, el mundo en el que vivimos, su situación y sus posibilidades (2009, p. 32). Por lo tanto, no se trata de prescribir una definición del pensamiento que rija la aplicación de la propuesta: “qué significa pensar o cómo entenderlo es una cuestión filosófica $\mathrm{y}$, por tanto, algo que precisa ser trabajado en la propia práctica del filosofar" (Kohan, en Sánchez-Manjavacas, 2020, p. 113).

Frente a esta aproximación, la propuesta de $F p N$, al querer estructurar previa y detalladamente el proceso, trastornaría el carácter filosófico de la educación, especialmente, la posibilidad del docente de situarse dentro de la filosofía (Kohan, 2016b, pp. 68-69). La propuesta de FpN estaría yendo, así, contra el carácter esencialmente liberador de la filosofía:

La filosofía es una forma de afecto, de afectar y afectarse en el encuentro de dos cuerpos que piensan juntos. Por eso es también un ejercicio de libertad, porque en ella, en su experiencia, se es más de uno, por lo menos dos, y, quien sabe, muchos. [...] la filosofía en la escuela, en el encuentro con otros maestros y estudiantes, niños y adultos, nos torna más libres: porque nos permite salir del mundo individual, potenciando nuestra libertad de estar con otros en el mundo (Kohan y Olarieta, 2013, p. 42)

Retomando la idea de scholé, Kohan resalta que, desde su origen, la escuela ha sido pensada como una suspensión del tiempo cronológico y sus exigencias orientadas a la productividad, una suspensión, en general, de lo "natural" para revelarlo como construido, sedimentado, contingente, y por lo tanto, transformable; y su reemplazo por una temporalidad distinta, libre, intensiva, 
donde las desigualdades del mundo real son puestas entre paréntesis (Masschelein y Simons, 2014, p. 31; Kohan, 2009, pp. 18, 25; 2016, p. 98). La escuela debería ser, entonces, el espacio del tiempo libre, entendido como "tiempo liberado de la carga de que todo debe tener una finalidad, un producto, un objeto" (Skliar, 2017, pp. 27-28). Resalto, junto a Skliar, que esta detención del tiempo cronológico no implica un aislamiento, sino que reconoce la historicidad, tiene memoria, pero la concibe de forma dinámica y no estática (2017, p. 43).

\section{2 la infancia}

Para Kohan, hay que distinguir entre la infancia como una etapa cronológica de la vida y la infancia como una posibilidad de la experiencia o modo de vivir un tiempo no cronológico. Esta segunda sería una experiencia de apertura, de asombro ante el misterio mismo de la existencia, que propicia nuevos modos de pensar, sentir y actuar (Kohan, 2016, p. 113; 2009: 25; Santiago, 2006, pp. 33-35; Wozniak, 2013, pp. 127-128, 133). Esta experiencia no puede restringirse a una edad ni un espacio institucionalizado: los niños no siempre viven su infancia en sus primeros años, dentro de la escuela y la condición del adulto no es siempre opuesta a la del niño. La infancia así entendida es la "condición de posibilidad de la existencia humana", una forma de experimentar la vida que nos acompaña permanentemente y que puede ser reactivada en espacios y momentos específicos (Kohan 2004, p. 273; 2009, p. 15). Se caracteriza por una vulnerabilidad, "una condición de estar afectado", entregado al mundo y a los otros (Kohan, 2004, pp. 267-268, 270). Así, la infancia sería nuestra condición finita y abierta, esto es, inacabada: no se trata de educarnos para superarla, sino recuperarla; en esto consistiría el carácter emancipador de la educación y su vínculo con la política (Kohan, 2004, pp. 275-276, 278-279).

Entonces, antes de ser una etapa específica de la vida (la inicial), la infancia es un destino (Skliar, 2017, p. 65). Esto se asemeja al planteamiento de Nietzsche en Así hablo Zaratustra, en tanto "la infancia está al final y no al principio: es la infancia, en última instancia, lo que da sentido a todas las otras transformaciones" (Kohan, 2009, p. 102). Ciertamente, este no es un objetivo a lograr de la misma calidad que los objetivos planteados en programas educativos 
a la $F p N$, sino que Kohan reconoce su carácter utópico, lo cual no le resta su rol como ideal que orienta la propuesta (2009, p. 115; 2004, pp. 274, 276).

\section{4. algunas críticas desde fcn a fpn}

\section{1 sobre el método y el curriculum}

Uno de los puntos que marcan la distancia entre la propuesta de $F_{c} N$ y FpN es el método. Mientras Kohan sostiene que no hay un método, y, por lo tanto, su propuesta es a nivel procedimental mucho más flexible y sensible al contexto -al no seguir un programa tan estrictamente estructurado-, Lipman y Sharp defienden uno con principios y premisas específicas (Lipman, Sharp y Oscayan, 1992, p. 167; Lipman, 2003, pp. 48, 163, 172; Kohan, en Sánchez-Manjavacas, 2020, p. 113; Kohan, 2009, p. 63). Según Lipman, el proceso educativo es "una red de políticas, principios y procedimientos a los que apelamos cuando organizamos la estructura de este proceso" (Lipman, 2016, p. 15). Ahora, es cierto que, siguiendo a Dewey, dentro de $F p N$ se concibe el método (la forma) como inherentemente ligado al contenido (materia), por lo que sería injusto decir que $F p N$ es una propuesta puramente procedimental (Lipman, Sharp y Oscanyan, 1992, p. 145; Sharp y Splitter, 1995, pp. 21, 43, 46; Dewey, 1938, pp. IV, 286). Sin embargo, FpN no deja de enfatizar la importancia del rigor del método sobre el contenido y la necesidad de un material con "modelos que les muestren [a los niños] cómo pensar" (Lipman, 2004, p. 133; Lipman, Sharp y Oscayan, 1992, p. 195).

Justamente, de este énfasis en el método parte la desconfianza de Lipman frente a la diversificación propuesta por la segunda generación. Por un lado, Murris critica esta postura en tanto cree que "el curriculum no es una condición necesaria ni suficiente para la enseñanza del pensamiento filosófico" (2016, p. 63). Es decir, la postulación de un método, objetivos y currículum para lograr determinados objetivos no garantiza que se genere una experiencia de investigación y pensamiento filosófico con los niños.

Por otro lado, el problema del método se relaciona al carácter instrumental otorgado a la filosofía dentro de la propuesta de $F p N$, el cual se vincula a la mentalidad del desarrollo. Según Vansieleghem, el método y la postulación de 
ideales regulativos como la democracia y razonabilidad, implica que "la autonomía que el niño gana mediante Filosofía para niños [...] no es más que la libertad para ocupar un lugar pre-constituido en tal discurso" (Vansieleghem, 2005, p. 25). De igual forma, Haynes y Murris argumentan que las preocupaciones instrumentales de $F p N$ "pueden desvirtuar las maneras en las que el pensamiento filosófico puede enriquecer y fortalecer toda la educación. Los objetivos obstaculizan la exploración filosófica autentica, y abierta" (Haynes y Murris, 2013, p. 289). Kennedy, por su lado, sostiene que "las metas implícitas de la educación [...] contradicen directamente un gran requisito para el diálogo -que solo es posible en un contexto de relaciones no instrumentales" (Kennedy, 2006, p. 166). Biesta, siguiendo esta línea, señala que el riesgo de la instrumentalización de la filosofía en estas propuestas es, precisamente, que se pierde el carácter propiamente filosófico al reducir el trabajo filosófico con niños al pensamiento crítico, el cual es considerado como una herramienta útil para navegar el mundo incierto del capitalismo (Biesta, 2017, p. 418). Es decir, la postulación de objetivos de desarrollo específicos reduce, si no es que anula, el potencial liberador de $F p N$.

De modo similar, Kohan y Costa sostienen que el problema recae en que la postulación de un método reduce las posibilidades de una auténtica investigación filosófica, cuando, por el contrario, lo propio de una genuina investigación filosófica está en la aceptación de la incertidumbre, la imposibilidad de una completa anticipación de sus caminos y resultados (Kohan y Costa, 2019, pp. 287-288). Debido a esto, el énfasis de los defensores de $F c N$ en el proceso por sobre tales objetivos no implica una anulación de los resultados, sino, simplemente, el reconocimiento de que estos son "inesperados, provisionales y creativos" (Murris, 2008, p. 675).

Además, según las propuestas de $F c N$, el programa de $F p N$ (esto es, los textos y los planes discusión que los acompañan) cargan con presupuestos que pueden condicionar la relación de los alumnos con los temas presentados e impedir una genuina exploración filosófica (Kohan y Waksman, 1999, p. 45). Como bien señala Murris: "La selección de un texto sobre otro es también normativa, en tanto prescribe lo que la filosofía es" (Murris, 2016, p. 68). Así, la 
selección del material no es neutral ni universal como se pretende, ya que refleja qué problemas, posturas, autores y tradiciones se consideran relevantes dentro de la historia de la filosofía y, consecuentemente, una concepción específica de la misma y su práctica. En el caso de $F p N$, deja de lado autores y temas exteriores a la tradición occidental-anglosajona. El simplemente designar un material a los maestros oculta el carácter cuestionable de esta selección (Kohan y Waksman, 1992, pp. 45-49). Asimismo, se podría decir que estos textos y guías, diseñados hace más de cuarenta años, no contemplan desarrollos filosóficos actuales y pueden parecer desfasados frente a nuestra coyuntura en constante y acelerado cambio (Kohan y Waksman, 1999, pp. 45, 48-49). Además, existe el problema secundario de la traducción de los materiales, que nos enfrenta a la disyuntiva entre mantenernos fieles al sentido del texto o a los problemas filosóficos y el objetivo del mismo (Kohan y Waksman, 1999, pp. 27, 39-40).

Como se puede observar, esta crítica se relaciona a la consideración del docente y su papel en el proceso educativo. Según Kohan, la insistencia en un método definido disminuye el rol del docente en el proceso educativo, y esto es particularmente grave en el caso de la filosofía, ya que “la selección del contenido filosófico explícita o implícitamente contenido dentro del material educativo para enseñar filosofía posiciona al maestro dentro de la tradición filosófica", lo cual no es necesariamente algo fácil de identificar, especialmente en el caso de profesores no formados en filosofía (Murris, 2016, p. 68). Según Kohan, el problema no está en la real capacidad de los maestros, sino en la actitud asumida hacia ellos: "[...] el que impone un método precisa presuponer que el o la maestra no pueden encontrar el mejor método", mas esto no se debe a que "el o la maestra no pueden encontrar uno que ellos se lo ofrecen", sino que "es como no tomarse a los docentes en serio, como subestimarlos, como si hubiera el gran o la gran filósofa que sabe cómo hacerlo y los que necesitan de la guía de aquel porque no pueden hacerlo por sí mismos" (Kohan, en Sánchez-Manjavacas, 2020, pp. 120, 113). Por el contrario, la renuncia al método, el aparato de objetivos, metas, reglas, e ideas preconcebidas, puede tener mayores alcances: "asumiendo que no hay un método para pensar, solo podemos interpretar la filosofía para niños como un regalo, algo excepcional, extraordinario" (Vansieleghem, 2005, p. 33). 
Ahora, si bien las propuestas de $F c N$ están libres de ciertos presupuestos de la propuesta de $F p N$ relacionados al contexto americano y pragmatista en el que se inserta -esto es, el protagonismo de los ideales de razonabilidad y democracia- también tienen los propios, específicamente, respecto al carácter político otorgado tanto a la educación como a la filosofía, además de la mencionada negación de la necesidad de un método (Kohan, 2009, pp. 63, 65, 68). Por ejemplo, uno de los principios de Kohan se relaciona a la importancia de la originalidad, retomando la idea de Simón Rodríguez resumida en la disyunción "inventamos o erramos", según la cual, debido a la absoluta novedad que representa Latinoamérica, es necesario que inventemos nuestras propias propuestas educativas, en vez de simplemente imitar modelos europeos (Kohan y Olarieta, 2013, pp. 37 ss.). En esa línea, la mera aplicación de un programa prediseñado implicaría entender la educación como un proceso mimético anulando su potencial emancipador-, en el que se buscaría un otro externo al cual parecerse, alejando a los maestros del proceso de creación de los materiales y perdiendo otras alternativas interesantes vinculadas a los contextos propios (Kohan, 2009, 64).

Como se mencionó antes, ambas propuestas mantienen la dinámica principal de la comunidad de investigación dialógica, donde la discusión sigue la guía de los intereses, dudas y reflexiones de los involucrados, y el sentido es constituido dialógica y cooperativamente (Kohan, 2010, p. 62). No obstante, es importante resaltar que desde $F_{c} N$ se defiende una lectura no metódica de la comunidad de investigación, para evitar el riesgo de instrumentalización descrito por Biesta, esto es, "transformar la práctica de la comunidad de investigación en una aproximación técnica, y, así, instrumentalizar el proceso (Kohan y Costa, 2019, p. 278). Esta propuesta considera que la comunidad ha de centrarse en "la experiencia de la presencia del otro", en vez del objetivo de desarrollar un set de habilidades (Vansieleghem, 2005, p. 33). De esta manera, FcN también considera que la forma del diálogo es importante y está inherentemente ligado al contenido del mismo, pero el diálogo filosófico se entiende de forma más amplia que en $F p N$, “como la explicitación y comprensión de los desacuerdos", es decir, no tiene un fin específico predelineado, como el 
consenso, resolución de problemas o desarrollo de habilidades determinadas, sino que se concentra en la exposición y comprensión profunda de las opiniones, propias y ajenas, para pensar colectivamente en la realidad (Kohan y Waksman, 1992, p. 71; Kohan y Olarieta, 2013, pp. 25, 30; Wozniak, 2013, p. 134).

Kohan sugiere como una posibilidad para plantear esta experiencia cinco momentos de composición de una clase: la disposición inicial, la vivencia, la problematización, la elección de temas a investigar, el diálogo, y una fase de evaluación, entendida como la valoración de la experiencia (Kohan y Olarieta, 2013, pp. 24-25). No obstante, Kohan advierte que esta sugerencia no es una suerte de "receta" a seguir, sino "una materia plástica, para que sobre ella se comience a componer la obra propia", y por eso, los docentes han de mantenerse atentos a lo que acontece en la experiencia y abiertos a hacer las modificaciones correspondientes, de manera que el no cumplimiento de la composición no sea visto como algo necesariamente malo (Kohan y Olarieta, 2013, p. 24).

\section{2 el papel del docente}

Esta flexibilidad y énfasis en la originalidad resalta en la asignación de la responsabilidad de la composición de las experiencias con los niños a cada uno de los docentes involucrados en la puesta en práctica de $F_{c} N$. "Partimos de que todxs pensamos y podemos igualmente aprender cualquier cosa si se nos dan las condiciones para hacerlo, que docentes son filósofos y filósofas que piensan y deciden cómo relacionarse con la filosofía y cómo propiciar condiciones para que los infantes de diversas edades se encuentren con ella" (Kohan, en SánchezManjavacas, 2020, pp. 112-113). De ahí que los maestros puedan y deban elegir libremente el material de estímulo o textualización, siempre y cuando se haga un uso crítico del mismo, este propicie aquella apertura a la experiencia y tenga una buena calidad, es decir, que su valor no se reduzca a lo meramente instrumental (Kohan y Waksman, 1999, p. 47). Este aspecto es central, ya que no es propio del filósofo ni del docente dejar la responsabilidad de la toma de decisiones sobre su propia práctica a un método creado por terceros.

Contrario a lo que propone $F p N$, desde $F c N$ se concibe que el ejercicio del docente ha de ser reflexivo. Siguiendo a Deleuze, Kohan defiende que el rol de 
los docentes no se reduce a la educación del otro, sino que es un proceso autorreflexivo y autotransformador, un "tornarse niño a través del acto de educar" (Deleuze, en Kohan, 2004, p. 283). El énfasis, como en Freire, no está en la adquisición de herramientas o técnicas, sino en este ejercicio de la reflexión, el cual ya contiene un potencial político (Kohan y Waksman, 1999, p. 18). Por eso, “El profesor no está para explicar lo que sabe ni el estudiante para aceptar el camino señalado por el profesor - cada uno debe buscar y encontrar, por sí mismo, con la compañía de los otros, su propio camino" (Kohan y Olarieta, 2013, p. 39).

Esto no quiere decir que el papel del maestro y el alumno se identifiquen, sino que el del primero se reconfigura: "El profesor pasa a cuidar de la manera en que el estudiante quiere aprender. Quiere que el estudiante quiera aprender, que busque, que nunca deje de buscar" (Kohan y Olarieta, 2013, p. 40). Entonces, dentro de $F c N$, la relación maestro-alumno es una de mutuo aprendizaje, es decir, el maestro no es un mediador entre la filosofía y el niño y no se asume que el maestro provee algo al niño unidireccionalmente: "tal vez enseñar no quiere decir transmitir a otros lo que alguien sabe -transmitir lo que se sabe a alguien que no lo sabe-, sino desde el no saber, o en todo caso sabiendo que no se sabe, ayudar al que sabe a cambiar de relación con el saber" (Kohan, 2016b, p. 156). Kohan es muy claro en este aspecto: "lo que nosotros queremos es que los docentes se sientan filósofos, vivan la filosofía [...] porque es eso lo que un niño o niña aprende de un docente: su relación con lo que hace, la manera en que lo vive. [...] se trata de crear las condiciones para vivir filosóficamente, maestra y estudiantes" (Kohan, en Sánchez-Manjavacas, 2020, p. 121). Los mismos docentes involucrados en la puesta en práctica de esta propuesta reconocen en sus testimonios el valor especial de estas experiencias: la apertura de espacios de reflexión filosófica les permite reflexionar críticamente sobre su práctica, no solo cuando dirigen experiencias filosóficas, sino también en otras situaciones educativas (Wozniak, 2013, pp. 129 ss.; Kohan, 2009, pp. 67 ss.) ${ }^{3}$.

\footnotetext{
3 "Es tan lindo sentir lo que se genera en los y las docentes cuando perciben que son ellos (y ellas) los que deciden cómo trabajar, con qué. [...] Cuando despliegas una relación filosófica con el enseñar y el aprender y los modos de ejercitarlos y no tiene que aplicar la filosofía del otro cuando no les impones tu manera de hacer las cosas, sino que les propones acompañarles en la manera
} 
Como ya se ha mencionado, vivir la filosofía como experiencia de pensamiento también implica la problematización de "cómo disponer la filosofía o el filosofar" (Kohan, en Sánchez-Manjavacas, 2020, p. 113), esto es la determinación de las formas, la selección de materiales, y "si el docente no se compromete con el filosofar, si no participa activamente y desde dentro del pensar que allí está teniendo lugar, si no filosofa, o por lo menos intenta hacerlo, entonces difícilmente podrá propiciarlo en los niños" (Kohan, 2009, p. 81). Según Kohan, este aspecto cuestionador y autorreflexivo es anulado en $F p N$ : “ ¿si el sentido de un programa de $F p N$ fuera un docente realmente crítico, no significaría la propia muerte de este programa? ¿Qué docente realmente crítico estaría contento en aplicar un programa que ya le viene dado de antemano?" (Kohan, en Sánchez-Manjavacas, 2020, p. 116).

\section{4 la dimensión política}

Por último, sobre la relación entre la política, la filosofía y la educación, Kohan sugiere que el objetivo de Lipman y Sharp de formar valores y ciudadanos democráticos es problemático porque (1) no todo el mundo está regido por tales sistemas, limitando la aplicabilidad de la propuesta y (2) la democracia -como cualquier otro sistema concreto, ideología, creencia o estado de cosas- ha de ser objeto de cuestionamiento de la filosofía, no su presupuesto (Kohan y Waksman 1999: 5; Kohan 2009: 105, 107). Efectivamente, los ideales defendidos por el programa de $F p N$ parecen determinados por las convicciones de sus autores antes que basarse en la naturaleza del ejercicio filosófico-educativo-infantil. Shapiro asegura sobre Lipman que "llegado a cierto punto en sus reflexiones, su criticidad alcanza un límite y su visión se percibe muy marcada por su historia como ciudadano estadounidense durante el siglo $\mathrm{XX}$, atravesado por el macartismo, la Segunda Guerra Mundial, la Guerra de Vietnam, la Crisis de los Misiles. [...] en Lipman-en-tanto-ciudadano-estadounidense, se perfila un fuerte espíritu liberal y antisoviético" (2019, p. 3).

que van afirmando de relacionarse con lo que hacen" (Kohan, en Sánchez-Manjavacas, 2020, p. 120). 
Hay que aclarar que $F c N$ no defiende un ejercicio apolítico de la filosofía ni la educación. Por el contrario, reconoce que el vínculo entre filosofía y educación también involucra a la política, en tanto la filosofía constituye una forma de poder cuestionador frente al poder afirmativo y determinante de aquella (Kohan y Waksman, 1999, p. 25). “Una experiencia de pensamiento filosófico nos deja esa rara y extraordinaria sensación de que ya no pensamos de la misma manera, [....] de que la vida ya no puede ser vivida como lo era. [...] transforma la relación que mantenemos con lo que pensamos, con la vida que llevamos" (Kohan, 2009, p. 67). Como sostiene Skliar, la educación es (o debería ser) "el gesto de ir 'contra el orden natural de las cosas'” (2017, p. 28), un ejercicio de desnaturalización, desfamiliarización, asombro: ahí reside su carácter filosófico (Wozniak, 2013, pp. 124-125, 129). En esa línea, Biesta, por ejemplo, resalta nuestra capacidad de cuestionar si nuestro ambiente es uno al que vale la pena adaptarnos, frente a los mecanismos de adaptación inteligente que, aunque similares a los humanos en otros aspectos, carecen de esta (Biesta, 2017, p. 433).

Kohan es bastante crítico frente a $F p N$ en este punto, debido al discurso político que suscribe y sus consecuencias prácticas. Según este, el atractivo de $F p N$, expresado en su inclusión en "varias reformas educativas" recae que se trata de "una buena manera de formar ciudadanos competentes, críticos, creativos, en fin, ponele [sic] los nombres de orden que te gusten menos revolucionarios, subversivos, anarquistas, programa moralizantes, el 'buen pensar' ciudadanos capaces de un 'pensamiento de alto orden' moralmente autónomos [...] ¿Alguien puede creer que eso va a transformar las sociedades horrendamente excluyentes, injustas, antidemocráticas que vivimos? [...]" (Kohan, en Sánchez-Manjavacas, 2020, pp. 115-116). Es decir, FpN no contribuye a la transformación de la sociedad, sino que, por el contrario, sirve para normalizar su modelo injusto.

De forma similar, como se adelantó, Vansieleghem sostiene que "Filosofía para niños no puede ser una base para la democracia y libertad simplemente porque está determinada por un tipo específico de pensamiento y actuar de acuerdo con roles que se supone debemos satisfacer: esto es, ser ciudadanos autónomos, críticos, creativos y comunicativos. Las otras posibilidades son excluidas" (Vansieleghem, 2005, p. 20). FpN hace del pensamiento 
multidimensional el único principio organizador de la democracia y libertad, no obstante, el énfasis en las metas futuras del programa que predeterminan toda acción y pensamiento hace que estos no puedan ser verdaderamente libres, ya que se espera que los niños solo "ocupen un lugar pre-constituido en aquel discurso" (Vansieleghem, 2005, p. 25).

Asimismo, se considera que el vínculo entre política e infancia no es algo impuesto artificialmente, sino que: “Las infancias participan de la política, ayudémoslas o no a que lo perciban. No somos nosotros que las vamos a hacer participar de la política. Lo que podemos es ayudarlas a notar que su vida es política como la de todos los seres humanos y que si lo perciben y lo quieren problematizar con otros puede volverse una dimensión más interesante" (Kohan, en Sánchez-Manjavacas, 2020, p. 117). La infancia no está alejada de la política y, por lo tanto, unirlas no es un objetivo a lograr: lo que busca $F_{c} N$ es simplemente explicitar y explorar tal relación.

\section{conclusión}

Después de haber revisado ambas propuestas, sostengo que si bien, comparten una intención similar, a saber, introducir la filosofía como praxis en la escuela desde la infancia, se diferencian en la forma de llevar a cabo este cometido. La propuesta de Lipman y Sharp $(F p N)$ está pensada, originalmente, para el sistema educativo institucionalizado norteamericano o, por lo menos, uno a su estilo, por ende, justifica la introducción de la filosofía a la educación en general por su valor instrumental y se preocupa, principalmente, por el diseño del programa: establecer objetivos claros, herramientas para lograrlos y evaluar el proceso. De ahí su énfasis en la rigurosidad del método, el cual, ineludiblemente, minimiza el carácter creativo y filosófico de la práctica del docente.

Desde tal perspectiva, las propuestas de $F_{c} N$ y su énfasis en la apertura, flexibilidad e invención, son criticadas porque no garantizan la obtención de objetivos predefinidos. Es más, su insistencia en la imposibilidad de la anticipación es contraria a la idea misma de un "proyecto", tal cual como es entendida en la gestión, que está básicamente pensada desde la teoría de 
administración (management). Asimismo, se critica su falta de un programa, en tanto dificultaría demasiado la labor de los maestros, quienes se sentirían perdidos o que la propuesta es demasiado idealista, romántica, y por ende, imposible de aplicar.

Kohan responde a estas críticas señalando que la contraprueba está en la práctica, en las experiencias exitosas de la propuesta y los docentes que han pasado por experiencias de formación y se han tornado "extraordinarios filósofos y filósofas y propiciadores en otros de la filosofía" (Kohan en SánchezManjavacas, 2020, pp. 116-117). Estoy de acuerdo con esto, ya que es cierto que FcN tiene muchos seguidores en distintas partes del mundo y hay un historial de experiencias exitosas. De hecho, si consideramos la propuesta de $F c N$ en sus propios términos, la falta de los elementos característicos de un proyecto educativo no es un problema, sino el corazón de la misma: ser una respuesta cuestionadora desde la educación frente al orden capitalista preestablecido.

Las críticas que Kohan sí considera legítimas se relacionan a la escucha genuina de los docentes y niños involucrados en las experiencias filosóficas, la tarea de mantenerse fiel a la filosofía e infancia -en los sentidos amplios, abiertos y prácticos que hemos descrito-, en vez de una noción específica y exclusiva de las mismas (Kohan en Sánchez- Manjavacas, 2020, pp. 117, 121). Asimismo, Haynes y Murris señalan que " $F c N$ debe tomar en cuenta la inequidad e injusticia que condicionan la participación de los niños y jóvenes en el diálogo en el aula para ser considerada una pedagogía democrática, tiene que reconocer sus propias limitaciones" (Haynes y Murris, 2013, p. 287). Esto es, más allá de las intenciones del programa respecto a la relación simétrica entre estudiantes y maestros, no se debe perder de vista que estas relaciones están, de hecho, atravesadas por relaciones de poder, enmarcadas en un contexto social específico. Respecto a esto, creo que es verdad que uno de los retos de $F c N$ es mantener un diálogo abierto con sus participantes el cual requiere mantenerse atento a las dinámicas de poder existentes, y no negarlas al anteponer la premisa de la igualdad (Skliar, 2017, p. 37).

En resumen, lo que aleja a las propuestas de $F p N$ y $F c N$ son las premisas fuertes de la propuesta de Lipman y Sharp sobre el método y la obtención de 
resultados específicos, a saber, el desarrollo del pensamiento multidimensional y su subscripción a los ideales de democracia y razonabilidad, así como su consideración reducida sobre el papel del docente. Por eso, considero que las propuestas de $F_{c} N$ no solo son más interesantes sino que cuidan mejor el carácter filosófico y educativo de la experiencia de hacer filosofía con niños.

En la práctica, podemos ver distintos casos de aplicación de una u la otra propuesta, así como diferentes combinaciones de las mismas. De ahí que muchos descarten la separación nominativa y hablen de "Filosofía para/con Niños" ( $F p / c N$ o simplemente $F p c N)$. Kohan señala: “[...] cuando miro algunas prácticas que llevan el nombre de $F p N$ siento que tenemos poco en común, casi nada... y otras veces, siento mucha comunidad con prácticas que se llaman muy diferentemente [...] La cuestión, entonces, parece tener que ver con la importancia que le damos a los nombres y cómo llamamos lo que hacemos... ¿los nombres crean comunidad?" (Kohan, en Sánchez-Manjavacas, 2020, p. 112). Considero que ambos proyectos pueden entrar en un diálogo crítico y fomentar nuevas ideas y experiencias de pensamiento filosófico para, con, desde, en, durante y mediante la infancia.

Asimismo, comparto el énfasis en la experiencia por sobre los objetivos de la propuesta de $F_{c N}$, su consideración del papel de los maestros y el vínculo entre filosofía y política. Contra las objeciones que alegan que la introducción de la filosofía en la educación implica necesariamente un adoctrinamiento, sostengo que advertir que el elemento político no puede ser eliminado permite reconocer que siempre puede ser cuestionado y que, por lo tanto, debemos mantenernos atentos para no tomarlo como natural o estático. El cuestionamiento es precisamente lo que posibilita la transformación de lo dado, y es aquello que caracteriza al ejercicio filosófico. Hacer filosofía con niños es una de esas experiencias que no puede anticiparse por completo: para conservar su carácter filosófico, educativo e infantil, es necesario que nos aproximemos a ella con apertura y atención, con la disposición para dejarnos afectar por ella.

referencias

Cavell, S. (1979). The Claim of Reason. Oxford. Oxford University Press. 
Biesta, G. (2017). Touching the Soul? Exploring an Alternative Outlook for Philosophical Work with Children and Young People. Childhood \& Philosophy 13(28), 415-452.

Dewey, J. (1938). Logic. The theory of inquiry. Nueva York. Henry Holt and Company.

Haynes, J. y Murris, K. (2011). The Provocation of an Epistemological Shift in Teacher Education through Philosophy with Children. Journal of Philosophy of Education 45(2), 285-303.

Kennedy, D. (2006). The Well of Being: Childhood, Subjectivity and Education. Nueva York. State University Of New York Press.

Kohan, W. (2004). Infancia. Entre educación y filosofía. Barcelona. Laertes.

Kohan, W. (2009). Infancia y filosofía. México D.F. Progreso.

Kohan, W. (2010). Filosofía y educación. Caracas. Fundación para la cultura y las artes.

Kohan, W. (2016a). El maestro inventor: Simón Rodríguez. Caracas. Ediciones del Solar.

Kohan, W. (2016b). Viajar para vivir: ensayar. Buenos Aires. Miño y Dávila.

Kohan, W. (2020). Paulo Freire más que nunca: una biografía filosófica. Buenos Aires. CLACSO.

Kohan, W. y Costa, M. (2019). Finding Treasures: Is the Community of Philosophical Inquiry a Methodology?. Studies in Philosophy and Education 38, 275-289.

Kohan, W. y Olarieta, B. (Comps.) (2013). La escuela pública apuesta al pensamiento. Rosario. Homo Sapiens.

Kohan, W. y Waksman, V. (1999). Filosofía con niños. Buenos Aires. Novedades educativas.

Lago, J. C. (2006). Redescribiendo la comunidad de investigación. Madrid. Ediciones de la Torre.

Lipman, M. (2003). Thinking in Education. Cambridge. Cambridge University Press.

Lipman, M. (2004). Natasha: aprender a pensar con Vygotsky. Barcelona. Gedisa.

Lipman, M. (2008). Philosophy for Children's Debt to Dewey. In Pragmatism, Education and Children. International Philosophical Perspectives, en: Taylor, Michael, Schreier, Helmut, y Paulo Ghiraldelli (eds.). Pragmatism, Education, and Children: International Philosophical Perspectives, Amsterdam/New York: Rodopi, 143-152.

Lipman, M. (2016). El lugar del pensamiento en la educación. Barcelona. Octaedro.

Lipman, M., Sharp A., y Oscanyan, F. (1992). La filosofía en el aula. Madrid. Ediciones de la Torre.

Masschelein, J. y Simons, M. (2014). Defensa de la escuela. Buenos Aires. Miño y Dávila.

Matthews, G. (2014). El niño y la filosofía. México D.F. Fondo de Cultura Económica.

Murris, K. (2008). Philosophy with Children, the Stingray and the Educative Value of Disequilibrium. Journal of Philosophy of Education 42 (3-4), 667-685.

Murris, K. (2013). The Epistemic Challenge of Hearing Child's Voice. Studies in Philosophy and Education 32, 245-259

Murris, K. (2016). The Philosophy for Children Curriculum: Resisting “Teacher Proof" Texts and the Formation of the Ideal Philosopher Child. Studies in Philosophy and Education 35(1), 63-78.

Murris, K. (2018). Philosophy with Picturebooks. En M. Peters (Ed). Encyclopedia of Educational Philosophy (pp. 1877-1883). Singapur. Springer.

Murris, K. (2019). Children's Development, Capability Approaches and Postdevelopmental Child: The Birth to the Fourth Curriculum in South Africa. Global Studies of Childhood 9(1), 56-71.

Piaget, J. (1951). The Child's Conception of the World. Londres. Routledge.

Reed, Ronald F. y Johnson T. W. (1999). Friendship and Moral Education. Twin Pillars of Philosophy for Children. Nueva York. Peter Lang.

Sánchez-Manjavacas, J. (2020). Hacia la escucha con/entre la infancia. La posibilidad de entender el filosofar desde la filosofía con niños (FCN) (Entrevista a Walter Kohan). Pensar Juntos. Revista Iberoamericana de filosofía para niños 4, 109-126. 
Santiago, G. (2006). Filosofía, niños, escuela. Buenos Aires. Paidós.

Shapiro, C. (2019). El lugar de la deliberación en la filosofía para niños de Lipman. Childhood E Philosophy 15, 01-18.

Sharp, A. y Splitter, L. (1995). La otra educación. Buenos Aires. Manantial.

Skliar, C. (2017). Pedagogía de las diferencias. Buenos Aires. Noveduc.

Trombadori, D. (2010). Conversaciones con Foucault. Buenos Aires. Amorrortu Editores.

Vansieleghem, N. (2005). Philosophy for Children as the Wind of Thinking. Journal of Philosophy of Education 39(1), 19-35.

Vansieleghem, N. y Kennedy, D. (2011). What is Philosophy for Children, What is Philosophy with Children-After Matthew Lipman?. Journal of Philosophy of Education, 45 (2), 171-182.

Wozniak, J. (2013). Ejercicios para tornar el mundo extraño. En W. Kohan, y B. Olarieta (comps.). La escuela pública apuesta al pensamiento (pp. 117-140). Rosario. Homo Sapiens.

recibido en: 25.05 .2020

aprobado en: 29.06 .2020 